\title{
Comparison of Nutritional Status of School Children with and Without Mid Day Meal Programme
}

\author{
Savitri Byadagi (Corresponding author) \\ *Ph. D Scholar, Department of Food Science and Nutrition, \\ U.A.S, G.K.V.K, Bangalore 560065 \\ E-mail: shreya.byadagi8@gmail.com
}

Sunanda Sharan

Rtd. Professor, Department of Food Science and Nutrition,

U.A.S, G.K.V.K, Bangalore 560065

E-mail: sunandasharan1986@gmail.com

\author{
Madhusudan Nayak, C. \\ Dept. Agricultural Engineering, \\ U.A.S, G.K.V.K, Bangalore 560065 \\ E-mail: nayaka.nayakas.madhu@gmail.com
}

Received: June 4, 2015

doi:10.5296/jee.v6i1.7892
Accepted: June 20, 2015 Published: June 24, 2015

URL: http://dx.doi.org/10.5296/jee.v6i1.7892

\begin{abstract}
This cross sectional study was carried out to compare nutritional status amongst children of 14- 16 years age of three different sets of schools. Study was conducted at Private High School Campus (PHSC) without mid day meal programme, Govt. High School Hebbal (GHSH) with ISCKON mid day meal programme and Govt. High School Bashettahalli (GHSB) Doddaballapur, with Govt. mid day meal. Height, weight and body mass index (BMI) were measured. When height and weight of the study subjects compared with $50^{\text {th }}$ percentile values of IAP standards, majority of the subjects with no midday meal programme were
\end{abstract}




\section{Macrothink}

Journal of Environment and Ecology

ISSN 2157-6092 2015, Vol. 6, No. 1

found to have better height (18.5\%) compared to with MDM subjects. Whereas, majority of the subjects in PHSC (without MDM) and GHSH (with ISCKON MDM) were found to have on par results for weight at 18.75 and 19.18 per cent respectively. Comparison of BMI with $50^{\text {th }}$ percentile values of WHO standards projected that majority of the PHSC (no MDM) subjects (56.2\%) had normal BMI as compared to with MDM subjects as compared to other two school

Keywords: Nutritional status, Mid Day Meal, anthropometry, BMI, height and weight 


\section{Introduction}

Children are the wealth of any country. Special attention should be paid to meet the needs of this group, constituting one fifth of the country's population. The physical growth of children is reflected by different anthropometric measurements especially weight and height. The physical dimensions of the body are much influenced by nutrition in growing period of school age. Poor health and nutritional status will affect work capacity as well as cognitive functions. And it is this age group that is a dynamic period of growth and development as children undergo physical, mental, emotional and social changes. Hence, it is necessary to assess the nutritional status of this demographic group. There is limitation of work related to nutritional status of school children from different sets of schools, age group, sex and rural or urban settings, nutritional status could be affected by the food provision or meal provided by different organization. Hence the present investigation was undertaken with the objectives to assess the nutritional status of school children with and without mid day meal programme.

\section{Material and Methods}

A total of 1092 school children from three different schools formed the sample. The samples drawn were cross sectional and included both male and female subjects. Data collection was carried out based on random sampling procedure. The age was recorded as indicated in the school records. Height was measured using portable height rod and weight by personal weighing balance with minimum clothing. Height and weight Measurements were recorded and were compared with IAP standards. Whereas BMI was calculated and compared with WHO standards.

\section{Result and Discussion}

It can be seen from Table 1 that when each school taken as a whole the total results showed that higher per cent of subjects belonged to below the IAP standard in GHSH (92.29\%) and GHSB (91.21\%) with MDM as compared to PHSC (81.5\%) without MDM. Collectively overall results showed that majority of the subjects were nested below the standard at 89.01 per cent as compared to above the standard of 10.99 per cent. Chi-square when applied for age and gender combination; results showed that 15 (20.94) and 16 (9.57) year old subjects showed significant results in height at five per cent level for age wise distribution. Females (41.06) were able to show significant results at five per cent level as compared to males in gender wise distribution.

Table 1. Age and gender wise distribution of height of the subjects as per Indian Academy of Pediatrics (IAP) standard

\begin{tabular}{|c|c|c|c|c|c|c|c|c|c|c|}
\hline \multirow{2}{*}{$\begin{array}{c}\text { Age } \\
\text { (Years) }\end{array}$} & \multirow{2}{*}{ GenderPHSC without MDM (n=324) } & GHSH with ISKCON MDM (n=438) & \multicolumn{2}{|c|}{ GHSB with Govt. MDM (n=330) } & \multicolumn{2}{|c|}{ Total (N=1092) } & Chi Square \\
\cline { 2 - 10 } & & $<$ STD & $>$ STD & $<$ STD & $>$ STD & $<$ STD & $>$ STD & $<$ STD & $>$ STD & \\
\hline \multirow{2}{*}{$\mathbf{1 4}$} & $\mathbf{M}$ & $55(88.7)$ & $7(11.3)$ & $40(90.9)$ & $4(9.1)$ & $51(98.1)$ & $1(1.9)$ & $146(92.4)$ & $12(7.6)$ & $3.73 \mathrm{NS}$ \\
\cline { 2 - 11 } & $\mathbf{F}$ & $36(92.3)$ & $3(07.7)$ & $42(95.5)$ & $2(4.5)$ & $44(97.8)$ & $1(2.2)$ & $122(95.3)$ & $6(4.7)$ & $1.402 \mathrm{NS}$ \\
\hline \multirow{2}{*}{$\mathbf{1 5}$} & $\mathbf{M}$ & $59(90.8)$ & $6(09.2)$ & $97(93.3)$ & $7(6.7)$ & $62(93.9)$ & $4(6.1)$ & $218(92.8)$ & $17(7.2)$ & $0.561 \mathrm{NS}$ \\
\cline { 2 - 10 } & $\mathbf{F}$ & $33(61.1)$ & $21(38.9)$ & $102(93.6)$ & $7(6.4)$ & $62(88.6)$ & $8(11.4)$ & $197(84.5)$ & $36(15.5)$ & $30.377 *$ \\
\hline \multirow{2}{*}{$\mathbf{1 6}$} & $\mathbf{M}$ & $56(84.8)$ & $10(15.2)$ & $60(93.8)$ & $4(6.3)$ & $38(86.4)$ & $6(13.6)$ & $154(88.5)$ & $20(11.5)$ & $2.796 \mathrm{NS}$ \\
\hline
\end{tabular}




\begin{tabular}{|c|c|c|c|c|c|c|c|c|c|c|}
\hline & $\mathbf{F}$ & $25(65.8)$ & $13(34.2)$ & $66(90.4)$ & $7(9.6)$ & $44(83.0)$ & $9(17.0)$ & $135(82.3)$ & $29(17.7)$ & $10.435 *$ \\
\hline \multirow[t]{2}{*}{ Age } & 14 & $91(90.1)$ & $10(09.9)$ & $82(93.2)$ & $6(6.8)$ & $95(97.9)$ & $2(2.1)$ & $268(93.7)$ & $18(6.3)$ & $5.22 \mathrm{NS}$ \\
\hline & 15 & $92(77.3)$ & $27(22.7)$ & $199(93.4)$ & $14(6.6)$ & $124(91.2)$ & $12(8.8)$ & $415(88.7)$ & $53(11.3)$ & $20.94 *$ \\
\hline \multirow[t]{2}{*}{ Gender } & $\mathbf{M}$ & $170(88.1)$ & 23 (11.9) & $197(92.9)$ & $15(7.1)$ & $151(93.2)$ & $11(6.8)$ & $518(91.4)$ & $49(8.6)$ & $3.99 \mathrm{NS}$ \\
\hline & $\mathbf{F}$ & $94(71.8)$ & $37(28.2)$ & $210(92.9)$ & $16(7.1)$ & $150(89.3)$ & $18(10.7)$ & $454(86.5)$ & $71(13.5)$ & $41.06 *$ \\
\hline
\end{tabular}

PHSC - Private High School Campus, Hebbal, Bangalore

NS - Non significant

GHSH - Government High School Hebbal, Bangalore

* Significant at $5 \%$ level

GHSB - Government High School Bashettihalli, Doddaballapur

Among all three schools studied, overall results for weight showed that higher per cent of subjects in GHSB (86.87\%) with Govt. MDM belonged to below IAP standard as compared to other two schools. Overall, 82.42 per cent of the subjects found to be below the standard category whereas 17.58 per cent of the subjects were found to be above the standard value (Table 2).

Table 2. Age and gender wise distribution of weight of the subjects as per Indian Academy of Pediatrics (IAP) standards

\begin{tabular}{|c|c|c|c|c|c|c|c|c|c|c|}
\hline \multirow{3}{*}{\begin{tabular}{|c|} 
Age \\
(Years)
\end{tabular}} & \multirow{2}{*}{\multicolumn{3}{|c|}{$\begin{array}{c}\text { Gender PHSC without MDM } \\
(\mathrm{n}=324)\end{array}$}} & \multirow{2}{*}{\multicolumn{4}{|c|}{\begin{tabular}{|c|c|} 
GHSH with ISKCON MDM & GHSB with Govt. MDM \\
$(\mathbf{n}=\mathbf{4 3 8})$ & $(\mathrm{n}=330)$
\end{tabular}}} & \multirow{2}{*}{\multicolumn{2}{|c|}{$\begin{array}{c}\text { Total } \\
(\mathrm{N}=1092)\end{array}$}} & \multirow[t]{3}{*}{ Chi Square } \\
\hline & & & & & & & & & & \\
\hline & & $<$ STD & $>$ STD & $<$ STD & $>$ STD & $<$ STD & $>$ STD & $<$ STD & $>$ STD & \\
\hline \multirow[t]{2}{*}{14} & M & $52(83.9)$ & $10(16.1)$ & $35(79.5)$ & $9(20.5)$ & $45(86.5)$ & $7(13.5)$ & $132(83.5)$ & $26(16.5)$ & $0.856 \mathrm{NS}$ \\
\hline & $\mathbf{F}$ & $26(66.7)$ & $13(33.3)$ & 39 (88.6) & $5(11.4)$ & $39(86.7)$ & $6(13.3)$ & $104(81.3)$ & 24 (18.8) & $7.887 *$ \\
\hline \multirow[t]{2}{*}{15} & $\mathbf{M}$ & $56(86.2)$ & $9(13.8)$ & 87 (83.7) & $17(16.3)$ & $63(95.5)$ & $3(4.5)$ & \begin{tabular}{|l|}
$206(87.7)$ \\
\end{tabular} & 29 (12.3) & $5.386 \mathrm{NS}$ \\
\hline & $\mathbf{F}$ & $38(70.4)$ & $16(29.6)$ & $85(78.0)$ & $24(22.0)$ & $56(80.0)$ & $14(20.0)$ & \begin{tabular}{|l|}
$179(76.8)$ \\
\end{tabular} & 54 (23.2) & $1.742 \mathrm{NS}$ \\
\hline \multirow[t]{2}{*}{16} & M & $61(92.4)$ & $5(7.6)$ & $59(92.2)$ & $5(7.8)$ & $40(90.9)$ & $4(9.1)$ & $160(92.0)$ & $14(8.0)$ & $0.089 \mathrm{NS}$ \\
\hline & $\mathbf{F}$ & $27(71.1)$ & $11(28.9)$ & $49(67.1)$ & $24(32.9)$ & $43(81.1)$ & $10(18.9)$ & $119(72.6)$ & $45(27.4)$ & $3.083 \mathrm{NS}$ \\
\hline \multirow[t]{3}{*}{ Age } & 14 & $78(77.2)$ & $23(22.8)$ & $74(84.1)$ & $14(15.9)$ & 84 (86.6) & $13(13.4)$ & \begin{tabular}{|l|}
$236(82.5)$ \\
\end{tabular} & $50(17.5)$ & $3.23 \mathrm{NS}$ \\
\hline & 15 & $94(79.0)$ & $25(21.0)$ & $172(80.8)$ & $41(19.2)$ & $119(87.5)$ & $17(12.5)$ & $385(82.3)$ & 83 (17.7) & $3.76 \mathrm{NS}$ \\
\hline & 16 & $88(84.6)$ & $16(15.4)$ & $108(78.8)$ & $29(21.2)$ & $83(85.6)$ & \begin{tabular}{|l|}
$14(14.4)$ \\
\end{tabular} & \begin{tabular}{|l|}
$279(82.5)$ \\
\end{tabular} & 59 (17.5) & $2.24 \mathrm{NS}$ \\
\hline \multirow[t]{2}{*}{ Gender } & $\mathbf{M}$ & $169(87.6)$ & 24 (12.4) & $181(85.4)$ & 31 (14.6) & 148 (91.4) & $14(8.6)$ & $498(87.8)$ & 69 (12.2) & $3.09 \mathrm{NS}$ \\
\hline & $\mathbf{F}$ & $91(69.5)$ & $40(30.5)$ & $173(76.5)$ & $53(23.5)$ & $138(82.1)$ & \begin{tabular}{|l|}
$30(17.9)$ \\
\end{tabular} & 402 (76.6) & $123(23.4)$ & $6.54 *$ \\
\hline \begin{tabular}{|l|} 
Total \\
\end{tabular} & & $260(80.25)$ & 64 (19.75) & 354 (80.82) & $84(19.18)$ & $286(86.67)$ & 44 (13.33) & $900(82.42)$ & $192(17.58)$ & \\
\hline
\end{tabular}

PHSC - Private High School Campus, Hebbal, Bangalore

NS - Non significant

GHSH - Government High School Hebbal, Bangalore

* Significant at 5\% level

GHSB - Government High School Bashettihalli, Doddaballapur 
Table 3 revealed that Higher percentage of subjects were found to have normal BMI of 56.2 per cent in PSHC without MDM whereas, underweight (52.1\%) subjects were found to be in higher per cent in GHSB with Govt. MDM. While overweight $(22.1 \%)$ and obese $(4.1 \%)$ subjects were found to be higher percentage in GHSH subjects with ISCKON MDM as compared to other two schools.

Table 3. BMI Classification according to WHO standards

\begin{tabular}{|c|c|c|c|c|c|c|c|c|c|c|c|c|c|c|c|c|}
\hline \multirow{3}{*}{$\begin{array}{c}\text { BMI category } \\
\text { (WHO cut } \\
\text { off's) }\end{array}$} & \multicolumn{4}{|c|}{$\begin{array}{l}\text { PHSC without MDM } \\
(\mathbf{n}=\mathbf{3 2 4})\end{array}$} & \multicolumn{4}{|c|}{$\begin{array}{c}\text { GHSH with ISKCON MDM } \\
(\mathbf{n}=438)\end{array}$} & \multicolumn{4}{|c|}{$\begin{array}{l}\text { GHSB with Govt. MDM } \\
(\mathbf{n}=\mathbf{3 3 0})\end{array}$} & \multicolumn{3}{|c|}{$\begin{array}{c}\text { Total } \\
(\mathrm{N}=1092)\end{array}$} & \multirow[t]{2}{*}{$\begin{array}{c}\text { Grand } \\
\text { Total }\end{array}$} \\
\hline & $14 \mathrm{yrs}$ & $15 \mathrm{yrs}$ & $16 \mathrm{yrs}$ & Total & $14 \mathrm{yrs}$ & $15 \mathrm{yrs}$ & $16 \mathrm{yrs}$ & Total & $14 \mathrm{yrs}$ & $15 \mathrm{yrs}$ & $16 \mathrm{yr}$ & Total & $14 \mathrm{yrs}$ & $15 \mathrm{yrs}$ & $16 \mathrm{yrs}$ & \\
\hline & n $(\%)$ & $\mathrm{n}(\%)$ & n (\%) & n (\%) & $\mathrm{n}(\%)$ & n $(\%)$ & n (\%) & n (\%) & $\mathrm{n}(\%)$ & n (\%) & n (\%) & n $(\%)$ & n (\%) & n (\%) & $\mathrm{n}(\%)$ & n (\%) \\
\hline $\begin{array}{c}\text { Underweight } \\
\quad(<5 \text { th } \\
\text { percentile })\end{array}$ & $\begin{array}{c}40 \\
(39.6)\end{array}$ & $\begin{array}{c}43 \\
(36.1)\end{array}$ & $\begin{array}{c}40 \\
(38.5)\end{array}$ & $\begin{array}{c}123 \\
(38.0)\end{array}$ & $\begin{array}{c}28 \\
(31.8)\end{array}$ & $\begin{array}{c}64 \\
(30.0)\end{array}$ & $\begin{array}{c}47 \\
(34.3)\end{array}$ & $\begin{array}{c}139 \\
(31.7)\end{array}$ & $\begin{array}{c}53 \\
(54.6)\end{array}$ & $\begin{array}{c}70 \\
(51.5)\end{array}$ & $\begin{array}{c}49 \\
(50.5)\end{array}$ & $\begin{array}{c}172 \\
(52.1)\end{array}$ & $\begin{array}{c}121 \\
(42.3)\end{array}$ & $\begin{array}{c}177 \\
(37.8)\end{array}$ & $\begin{array}{c}136 \\
(40.2)\end{array}$ & $\begin{array}{c}434 \\
(39.7)\end{array}$ \\
\hline $\begin{array}{l}\text { Normal } \\
\text { (5th - 85th } \\
\text { percentile) }\end{array}$ & $\begin{array}{c}54 \\
(53.5)\end{array}$ & $\begin{array}{c}70 \\
(58.8)\end{array}$ & $\begin{array}{c}58 \\
(55.8)\end{array}$ & $\begin{array}{c}182 \\
(56.2)\end{array}$ & $\begin{array}{c}42 \\
(47.7)\end{array}$ & $\begin{array}{c}90 \\
(42.3)\end{array}$ & $\begin{array}{c}52 \\
(38.0)\end{array}$ & $\begin{array}{c}184 \\
(42.0)\end{array}$ & $\begin{array}{c}30 \\
(30.9)\end{array}$ & $\begin{array}{c}49 \\
(36.0)\end{array}$ & $\begin{array}{c}33 \\
(34.0)\end{array}$ & $\begin{array}{c}112 \\
(33.9)\end{array}$ & $\begin{array}{c}126 \\
(44.1)\end{array}$ & $\begin{array}{c}209 \\
(44.7)\end{array}$ & $\begin{array}{c}143 \\
(42.3)\end{array}$ & $\left|\begin{array}{c}478 \\
(43.8)\end{array}\right|$ \\
\hline $\begin{array}{l}\text { Over weight } \\
\text { (85th to } 95 \text { th } \\
\text { Percentile) } \\
\end{array}$ & $\begin{array}{c}7 \\
(6.9)\end{array}$ & $\begin{array}{c}6 \\
(5.0)\end{array}$ & $\begin{array}{c}5 \\
(4.8)\end{array}$ & $\begin{array}{c}18 \\
(5.6)\end{array}$ & $\begin{array}{c}17 \\
(19.3)\end{array}$ & $\begin{array}{c}50 \\
(23.5)\end{array}$ & $\begin{array}{c}30 \\
(21.9)\end{array}$ & $\begin{array}{c}97 \\
(22.1)\end{array}$ & $\begin{array}{c}11 \\
(11.3)\end{array}$ & $\begin{array}{c}17 \\
(12.5)\end{array}$ & $\begin{array}{c}13 \\
(13.4)\end{array}$ & $\begin{array}{c}41 \\
(12.4)\end{array}$ & $\begin{array}{c}35 \\
(12.2)\end{array}$ & $\begin{array}{c}73 \\
(15.6)\end{array}$ & $\begin{array}{c}48 \\
(14.2)\end{array}$ & $\begin{array}{c}156 \\
(14.3)\end{array}$ \\
\hline $\begin{array}{c}\text { Obese } \\
\begin{array}{c}(>95 \text { th } \\
\text { percentile) }\end{array}\end{array}$ & $\begin{array}{c}0 \\
(0.0)\end{array}$ & $\begin{array}{c}0 \\
(0.0)\end{array}$ & $\begin{array}{c}1 \\
(1.0)\end{array}$ & $\begin{array}{c}1 \\
(0.3)\end{array}$ & $\begin{array}{c}1 \\
(1.1)\end{array}$ & $\begin{array}{c}9 \\
(4.2)\end{array}$ & $\begin{array}{c}8 \\
(5.8)\end{array}$ & $\begin{array}{c}18 \\
(4.1)\end{array}$ & $\begin{array}{c}3 \\
(3.1)\end{array}$ & $\begin{array}{c}0 \\
(0.0)\end{array}$ & $\begin{array}{c}2 \\
(2.1)\end{array}$ & $\begin{array}{c}5 \\
(1.5)\end{array}$ & $\begin{array}{c}4 \\
(1.4)\end{array}$ & $\begin{array}{c}9 \\
(1.9)\end{array}$ & $\begin{array}{c}11 \\
(3.3)\end{array}$ & $\begin{array}{c}24 \\
(2.2)\end{array}$ \\
\hline
\end{tabular}

PHSC - Private High School Campus, Hebbal, Bangalore

GHSH - Government High School Hebbal, Bangalore

GHSB - Government High School Bashettihalli, Doddaballapur

The mean anthropometric measurements viz. height and weight were found to increase in the adolescent subjects with the successive increase in the age group. Similar findings were also obtained by [5] who observed that there was a linear increasing trend in height and weight of the study population whereas.

In the present study, the nutritional status of the study subjects was assessed by anthropometry. Results revealed that, height of subjects of three different schools (PSHC without MDM, GHSH with ISCKON MDM and GHSB with Govt. MDM programme) was significantly lower than the IAP standards irrespective of age and gender. [6] Reported similar results where, the mean heights of Akshara Dasoha beneficiaries in Karnataka were 10 per cent lower than NCHS standards irrespective of gender. The results are also on par with results of $[3,7]$ with respect to anthropometric measurements of school age (6-16 years) children. Similar findings were revealed by [1] and where MDM beneficiaries had lower height compared to non MDM subjects. 
Among all the three schools, majority of subjects of GHSB with MDM belonged to below the IAP $(86.87 \%)$ standard of weight as compared to other two schools.

Reason for variation in BMI of three schools could be due to the difference in their height and weight parameters. In PHSC without MDM most of the subjects had better height and weight as it has resulted in normal BMI, whereas GHSH (with ISCKON MDM) subjects mean height was less as compared to mean weight for their age these could have resulted in overweight and obese among these subjects. While GHSB subjects with Govt. MDM had lower mean height and weight which had resulted in underweight. [2] Observed in her study that there is a direct relationship between BMI and per cent body fat. Similar observations were reported by [4]. In the present study also similar trend was found in GHSH subjects as it has resulted in higher BMI (overweight and obese).

\section{Conclusion}

Based on the results of this study, it can be concluded that majority of the subjects from all the three schools belonged to below the defined anthropometric standards. However, comparatively PHSC without MDM subjects showed better nutritional status compared to schools with MDM.

Provision of MDM would have contributed to the improved nutritional status of under privileged population in the study group substantially. There is still a large gap to be filled towards better nutritional status by providing nutri rich meals.

\section{Reference}

Alim, F., Fhalil, S., Mirz, I., \& Khan, Z. (2012). Impact of mid-day meal scheme on the nutritional status and academic achievement of school children in Aligarh city. Indian J. Sci. Res. 3(2), 85-90

Bembem, K. (2010). Development of nutritious snack foods from indigenously processed grains for school children. Post Graduate M.Sc Thesis submitted to University of Agricultural Sciences, GKVK, Bengaluru.

Bharati, P., Itagi, S., \& Megeri, S. N. (2005). Anthropometric measurements of school children of Raichur (Karnataka). J. Hum. Ecol, 18(3), 177-179.

Choudhary, B., \& Kishore, A. (2004). Are we making school children sedentary and obese? Intervention study of 6000 Indian school children. The Ind. J. Nutr. Dietet, 41, 250-254.

Kochar, G. K., Neha, B., \& Maninder, K. (2010). Nutritional Status of Private And Government School Going Adolescent Males. Ind.J.Nutr.Dietet, 47, 533-538.

Naik, R. ( 2005). Evaluation of Akshara Dasoha Scheme of Karnataka. Dept. of Food Sci. and Nutr., Univ. Agric. Sci., Dharwad.

Nwokora, S. O., Ifada, K. O., \& Olomu, J. M. (2006) Anthropometric assessment of nutritional status and growth of 10-20 years old individuals in Benin city (Nigeria) Metropolis. Pak. J. Nutr.,5(2), 117-12. 


\section{Copyright Disclaimer}

Copyright for this article is retained by the author(s), with first publication rights granted to the journal.

This is an open-access article distributed under the terms and conditions of the Creative Commons Attribution license (http://creativecommons.org/licenses/by/3.0/). 